\title{
Reutilización y reciclaje de residuos sólidos en economías emergentes en Latinoamérica: una revisión sistemática
}

\author{
Alberto Quispe Palomino \\ qquispepa18@ucvvirtual.edu.pe \\ https://orcid.org/0000-0001-7754-7432 \\ Universidad Cesar Vallejo Lima-Perú \\ Vicente Quispe Huisa \\ vquispeh@ucvvirtual.edu.pe \\ Universidad Cesar Vallejo Lima-Perú
}

\section{RESUMEN}

Este trabajo de averiguación tiene como fin Detectar los hallazgos empíricos de la literatura de vanguardia sobre la reutilización y reciclaje de los residuos sólidos en las economías emergentes de América Latina en los años 2019-2020. El mismo que tiene un diseño de indagación de revisión bibliográfica sistemática, utilizando artículos científicos publicados en distintas bases electrónicas de revistas indexadas provenientes de los buscadores EBSCO, PROQUEST, SCOPUS, SCIELO y DIALNET, quedando seleccionados 12 artículos científicos, los cuales cumplen con las metas propuestas, después de utilizar los criterios de integración y exclusión siguiendo las directrices PRISMA. A partir del estudio de los resultados y las conclusiones logradas según las metas del presente análisis, se concluyó que las averiguaciones que más prevalecen fueron las de tipo básico, con enfoque cualitativo. Igualmente, es fundamental determinar que los estudios mencionados tienen interacción a las prioridades de la concepción de la reutilización y reciclaje de residuos sólidos con una orientación hacia la economía lineal. En conclusión, tenemos la posibilidad de asegurar que la reutilización y reciclaje de los residuos sólidos en América Latina todavía está en proceso de transición hacia una economía circular en la Administración de Residuos Rígidos.

Palabras clave: reutilización; reciclaje; economía lineal; economía circular. 


\title{
Solid waste reuse and recycling in emerging economies in latin america: a systematic review
}

\begin{abstract}
The purpose of this research work is to detect the empirical findings of the avant-garde literature on the reuse and recycling of solid waste in the emerging economies of Latin America in the years 2019-2020. It has a systematic bibliographic review inquiry design, using scientific articles published in different electronic databases of indexed journals from the EBSCO, PROQUEST, SCOPUS, SCIELO and DIALNET search engines, being selected 12 scientific articles, which meet the goals proposed, after using the integration and exclusion criteria following the PRISMA guidelines. From the study of the results and the conclusions reached according to the goals of this analysis, it was concluded that the most prevalent inquiries were those of a basic type, with a qualitative approach. Likewise, it is essential to determine that the aforementioned studies interact with the priorities of the conception of the reuse and recycling of solid waste with an orientation towards the linear economy. In conclusion, we have the possibility of ensuring that the reuse and recycling of solid waste in Latin America is still in the process of transition towards a circular economy in the Administration of Rigid Waste.
\end{abstract}

Keywords: reuse; recycling; linear economy; circular economy.

Artículo recibido: 02 noviembre. 2021 Aceptado para publicación: 28 noviembre 2021 Correspondencia: qquispepa18@ucvvirtual.edu.pe Conflictos de Interés: Ninguna que declarar 


\section{INTRODUCCIÓN}

En el planeta, las metrópolis generan alrededor de 1,3 mil millones de Tm. de desperdicios sólidos por lapso (1,2 kg/hab./día); se estima que este incrementará a 2,2 mil millones de Tm. (1,42 kg/ hab./día) para el año 2025. En América Latina y el Caribe, el promedio per cápita de desperdicios sólidos domiciliarios es de $0,6 \mathrm{~kg} / \mathrm{hab} /$ día y desperdicios sólidos urbanos de 0,9 kg/hab/día, el cual corresponde al $12 \%$ de la generación mundial de residuos municipales (160 millones de Tm.), distribuidos en residuos orgánicas (54\%), papel $(16 \%)$ y plástico $(12 \%)$, en otros términos producido gracias a la expansión de la urbanización, el crecimiento del consumo y los cambios de hábitos del estilos de vida de los habitantes (Graziani, 2018).

Latinoamérica y el Caribe es la zona más urbanizada de las naciones en desarrollo, con cerca de un $80 \%$ de su población viviendo en superficies urbanas, con una proyección para el año 2050 de 90\% (Rondón et al., 2016). Así mismo en dicha región, las Instituciones públicas y no públicas para la reutilización y el reciclaje de la Administración de Residuos Sólidos tuvieron problemas por ser actividades que generan varios costos y a la vez no son rentables; y su financiación es un reto a la suerte, así como el fortalecimiento de las instituciones y la consolidación de los inversionistas del sector privado; no obstante, la recuperación de precios es insuficiente, ya que las metrópolis logran cobrar a los ciudadanos (a través de tarifas municipales), a esto se añade el deficiente desempeño de recolección, en descomposición, de reciclaje de desechos y procedimiento térmico de desechos para la recuperación de energía, ocasionando inmensos focos de contaminación (Aire, Agua, Medio Ambiente) y patologías de todo tipo (Sánchez et al., 2020).

Otro de los problemas que se presenta es sobre la reutilización y reciclaje que se crean como parte del aprovechamiento de los residuos sólidos, para obtener materia prima, que sirva para incorporarla de forma directa a un proceso de producción o de consumo, con el propósito de obtener nuevos productos en una planta recicladora y que posibilite reducir la contaminación del aire, agua, suelo para vivir en un mundo independiente de contaminación (Sanmartin et al,. 2016). Por consiguiente actualmente la literatura de una economía circular en Latinoamérica y el Caribe, está dominada por prácticas industriales de procesos cerrados o modelo lineal de fabricación y consumo o sea los insumos se extraen, combinan, procesan, consumen y descartan (De La Cuesta y Pardo, 2020; Prieto, 
2018). Es decir el sistema lineal "tomar-hacer-desechar" fundamentalmente son orientados a políticas en la última fase del proceso lineal, por medio de la administración de residuos, el reciclaje y la reutilización (Yáñez, 2021; Patwa, 2021). A pesar de que la linealidad está profundamente institucionalizada, existe un interés creciente entre los productores, los gobiernos y los estudiosos en renunciar al modelo lineal por medio de la adopción de un modelo de economía circular, que permite consolidarse a las Instituciones y organizaciones con actividades económicas sustentables a fin de producir novedosas oportunidades empresariales innovadoras, eficientes con menos consumo; y construyendo puestos de trabajo a escala local adecuados para todos los niveles de habilidades (Romero y Rossi, 2017; Barros, 2021). Permitiendo ahorrar energía y ayudar a evadir los males irreversibles provocados en el clima, la diversidad biológica, la contaminación del aire, el suelo y el agua, la implementación de modelos de transición, innovación y tecnología, fortaleciendo la reutilización y reciclaje de desechos sólidos. (Goodwin et al., 2021; Ekins et al., 2019; Corona et al., 2019).

Además, Rincón et al. (2021); Tello P, Campani (2018) la Economía Circular, es la producción de bienes y servicios que se mantengan en el sistema económico en el lapso de tiempo más extenso, reduciendo el consumo y el desperdicio de materias primas, agua y fuentes de energía. Además, conocido como un nuevo modelo económico y social que tiene presente el medio ambiente y la sostenibilidad. Es por ello Rondón et al (2016) instituye que la administración de residuos sólidos dejará mantener el control del almacenamiento, la transferencia, el transporte, el procesamiento, el recojo, la generación y evacuación de residuos sólidos de manera transparente y ordenada con los mejores principios y expectativas de salud pública, estética, conservación, ingeniería y economía. Por otra parte según Turcott (2018) estable que los residuos municipales, son residuos provocados en los gobiernos centrales, regionales, locales, casas, organizaciones, negocio, oficinas administrativas, colegios, universidades y otras empresas donde se detectan residuos peligrosos como (pilas, recipientes, pintura, desinfectantes, insecticidas) y residuos no peligrosos como (papel, cartón, latas, recipientes de alimentos de vidrio, plástico, etc.). Así mismo, Tello y Campani (2018)

instituye que la valorización de los Residuos sólidos, se entiende como el proceso de cuantificar el precio importante de los diferentes recursos de los desperdicios sólidos, sean orgánicos e inorgánicos, en funcionalidad de su recuperación y aprovechamiento 
tendientes a reincorporar materiales a procesos productivos e industriales incluso de energía generada desde el procesamiento de los mismos.

Al final la administración integral de residuos sólidos urbanos constituye actualmente una inquietud trascendental por sus impactos directos e indirectos, irreversibles y permanentes en el medio ambiente y la salud pública (Rondón et al., 2016). Consecuentemente un camino hacia una economía circular es una probabilidad para modificar nuestra economía y hacerla más posible, para contribuir a las metas climáticos y la conservación de los recursos globales, que contribuirán a los Objetivo de Desarrollo Sustentable (Comision, 2018). Es así que, en los últimos años, el modelo de la economía circular ha recibido mucha atención y apoyo a un elevado grado político en Latinoamérica y el Caribe, con más de 80 iniciativas públicas en temas de economía circular. Esto involucra renunciar al modelo presente de economía lineal, fortaleciendo el modelo de economía circular en proceso de producción con bienes más duraderos y al mismo tiempo permita volver a usar, componer y reciclar los desechos sólidos (Schröder et al., 2020). Es por ello en el presente artículo de revisión sistemática se plantea las siguientes preguntas:

- ¿Qué metodología y herramientas de evaluación se utilizó?

- ¿Cuáles son las conclusiones y objetivos identificados sobre la reutilización y reciclaje de residuos sólidos?

- ¿Cuáles son los resultados sobre uso de Innovación y Tecnología en la reutilización y reciclaje de residuos sólidos?

Por consiguiente, dicho estudio se justifica bajo un nuevo modelo de economía circular, que implica cumplir con todos los procesos del manejo de los residuos sólidos como un todo, abordando la problemática ambiental de manera mucho más eficiente, proveyendo y reduciendo la minimización de impactos y residuos contaminados.

\section{ESTRATEGIAS METODOLOGICAS O MATERIALES O MÉTODOS}

En la presente investigación, se realizó una revisión sistemática que tuvo como unidad de análisis artículos originales y revisión, los cuales permitieron sintetizar y analizar la información científica recopilada de diferentes fuentes (Grijalva et al., 2019); para ello, es necesaria dejar claro el proceso y el método planificado para dicha revisión (Moher et al., 2016). Dicho análisis se llevó a cabo sobre "Gestión de Residuos Sólidos” a partir de los artículos publicados en los años 2017 al 2020 en las siguientes bases de datos: Ebsco, 
Proquest, Scopus, Scielo y Dialnet, el total de artículos encontrados fue de 260; de ellos, se descartaron aquellos que no contaban con los criterios de inclusión: estudios aplicados en universidades, estudios desarrollados en Latinoamérica, investigaciones aplicadas y artículos de acceso abierto. En cuanto a los criterios de exclusión se tomaron en cuenta los siguientes: estudios desarrollados en Gestión de Residuos Sólidos no científicos, investigaciones teóricas y artículos de discusión, investigaciones aplicadas no desarrolladas por universidades e investigaciones aplicadas fuera del contexto latinoamericano. Como resultado, de acuerdo con el método Prisma (Moher et al., 2016), se obtuvo un total de 12 artículos, los cuales cumplen con los objetivos propuestos y constituyen la base para la presente revisión.

\section{Figura 1}

\section{Proceso de Revisión PRISMA}

Índice de
Base de
Datos

Índice por nivel de Educación

Índice por región de análisis

Índice por Objetivos
Artículos registrados en base de datos (Ebsco, Pro Quest, Scielo y Dialnet) referidos a Gestión de Residuos Sólidos entre el 2017-2020 n=260

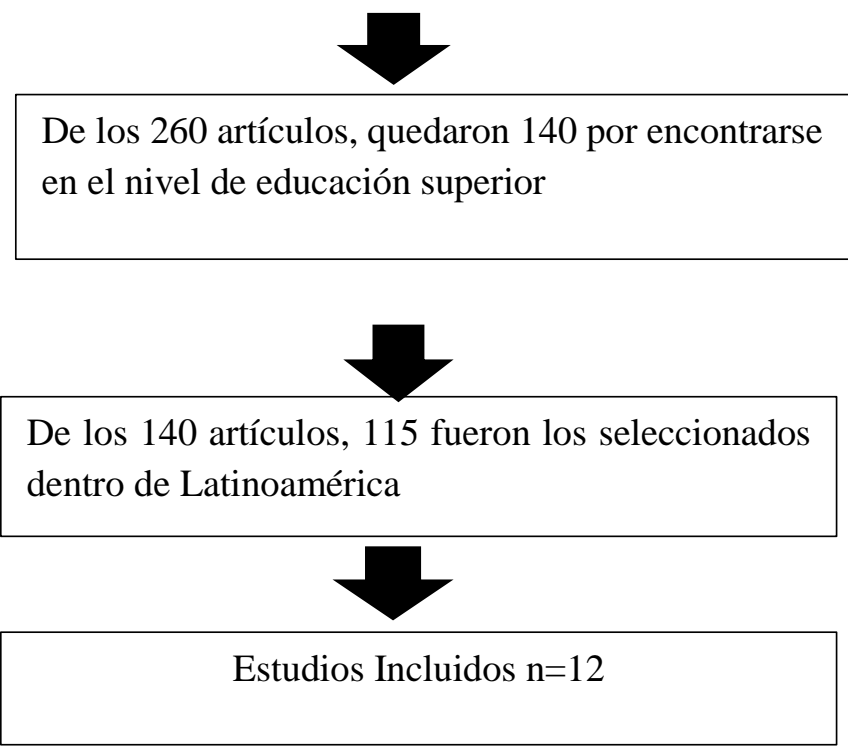

Nota. Se detalla el proceso de depuración de las referencias en las bases de datos siguiendo el modelo PRISMA 


\section{Tabla 1}

Resultados de selección en los repositorios.

\begin{tabular}{|c|c|c|c|c|c|c|c|c|c|}
\hline Repositorio & Código & $\begin{array}{l}\text { Combinación } \\
\text { de Búsqueda }\end{array}$ & Resultados & $\begin{array}{c}\text { Temática } \\
\text { (Estudios } \\
\text { Universit } \\
\text { arios) } \\
\end{array}$ & Tipo & Región & Acceso & Objetivo & $\begin{array}{c}\text { Artículo } \\
\text { Seleccionados }\end{array}$ \\
\hline EBSCO & A1 & $\begin{array}{ll}\text { Gestión } & \text { de } \\
\text { Residuos } & \\
\text { Solidos } & \end{array}$ & 81 & 11 & 11 & 11 & 11 & 2 & 2 \\
\hline $\begin{array}{l}\text { PROQUE } \\
\text { ST }\end{array}$ & B1 & $\begin{array}{ll}\text { Gestión } & \text { de } \\
\text { Residuos } & \\
\text { Solidos } & \end{array}$ & 42 & 8 & 8 & 8 & 8 & 1 & 1 \\
\hline SCOPUS & $\mathrm{C} 1$ & $\begin{array}{ll}\text { Gestión } & \text { de } \\
\text { Residuos } & \\
\text { Solidos } & \end{array}$ & 47 & 45 & 45 & 20 & 20 & 3 & 3 \\
\hline SCIELO & D1 & $\begin{array}{ll}\text { Gestión de } & \text { desiduos } \\
\text { Residos } & \\
\text { Solido }\end{array}$ & 13 & 10 & 10 & 10 & 10 & 2 & 2 \\
\hline DIALNET & E1 & $\begin{array}{ll}\text { Gestión de } & \text { desiduos } \\
\text { Residos } & \\
\text { Solido }\end{array}$ & 77 & 66 & 66 & 66 & 66 & 4 & 4 \\
\hline
\end{tabular}

En la tabla 1 se puede apreciar el proceso de selección; en función a los repositorios elegidos y el motor de búsqueda (que fue "Gestión de Residuos Sólidos") se pudieron obtener 260 artículos. Sin embargo, al tomar en cuenta el nivel de educación superior, la región (Latinoamericana), y su vinculación con los objetivos se seleccionaron solo 12 investigaciones.

\section{1.- Características de los artículos}

De acuerdo con el proceso de revisión Prisma, podemos observar que de las doce (12) fuentes seleccionadas en la presente revisión, la mayoría de trabajos publicados en Latinoamérica, se encuentran en Argentina y Ecuador regiones desde las cuales se han realizado tres (3) investigaciones en cada uno sobre el Reciclaje y Reutilización de residuos sólidos; le sigue Chile y Colombia con dos (2) trabajos publicados cada uno; y finalmente se encuentra Perú y Brasil, países en los que se ha desarrollado una investigación en cada región. 


\section{Tabla 2}

Artículos incluidos en la Revista

\begin{tabular}{|c|c|c|}
\hline Repositorio & Autor y año & Pais \\
\hline SCIELO & FilipaK, A; Stefanello, S; Okada, J; Huanzcker, M. 2020 & Brasil \\
\hline SCIELO & Moreno, K. 2019 & Perú \\
\hline DIALNET & Vélez, J. 2017 & Argentina \\
\hline DIALNET & Mendieta, R; Gilber, J; Menendes, C; Macías, R. 2020 & Venezuela \\
\hline DIALNET & $\begin{array}{l}\text { Herrera, I; Herrera, E; Collaguazo, G; Lorente, L; Saraguro, R. } \\
2018\end{array}$ & Ecuador \\
\hline EBSCO & Saidon, M. 2019 & Argentina \\
\hline EBSCO & Colorado, H; Echeverri, G. 2019 & Colombia \\
\hline EBSCO & Valenzuela, N. 2020 & Chile \\
\hline PROQUEST & Valderrama, C; Ortiz, H; Borrero, O. 2020 & Colombia \\
\hline PROQUEST & Vélez, A; Peñafiel, P; Heredia, M; Barreno, S; Chávez, J. 2019 & Ecuador \\
\hline PROQUEST & Villegas, F; Llonch, J; López, P; Valenzuela, L. 2021 & Chile \\
\hline SCOPUS & Maldovan, J. 2018 & Argentina \\
\hline
\end{tabular}

\section{Figura 2}

Porcentaje de fuentes de acuerdo con la Región de Procedencia

\section{Fuentes por Región}

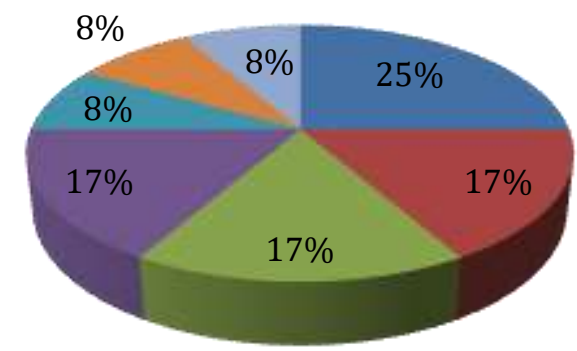

$\square$ Argentina $\square$ Ecuador $\square$ Colombia $\square$ Chile $\square$ Peru $\square$ Brasil $\square$ Venezuela

Nota. No se detalla el origen de los artículos por país

\section{RESULTADOS Y DISCUSIÓN}

En función al objetivo formulado, se expondrá los resultados de las investigaciones de los 12 artículos seleccionados.

\section{1- Metodología y Herramientas de Evaluación}

La revisión de las investigaciones nos revela que existe una preferencia respecto al enfoque metodológico. Podemos observar que, de acuerdo a lo declarado por los autores, 
existe una metodología que sobresalga respecto de las otras. Apreciamos que nueve (09) de las investigaciones optaron por un enfoque cualitativo y dos (2) por un enfoque cuantitativo. Solo uno (1) de las investigaciones se desarrolló con una metodología mixta.

\section{Tabla 3}

Metodología y Herramientas de Evaluación

\begin{tabular}{clcc}
\hline $\mathbf{N}^{\circ}$ & Autor y Año & Metodología & $\begin{array}{c}\text { Herramientas de } \\
\text { Evaluación }\end{array}$ \\
\hline 1 & FilipaK, A; Stefanello, S; Okada, J; Huanzcker, M. 2020 & Cualitativo & Entrevistas \\
\hline 2 & Moreno, K. 2019 & Cualitativo & Entrevistas \\
\hline 3 & Vélez, J. 2017 & Cualitativo & Entrevistas \\
\hline 4 & Mendieta, R; Gilber, J; Menendes, C; Macías, R. 2020 & Cualitativo & Encuetas \\
\hline 5 & Herrera, I; Herrera, E; Collaguazo, G; Lorente, L; Saraguro, R. & Cualitativo & $\begin{array}{c}\text { Análisis } \\
\text { Documental }\end{array}$ \\
\hline 6 & Saidon, M. 2019 & Cualitativo & $\begin{array}{c}\text { Análisis } \\
\text { Documental }\end{array}$ \\
\hline 7 & Colorado, H; Echeverri, G. 2019 & Cualitativo y & Encuetas \\
\hline 8 & Valenzuela, N. 2020 & Cuantitativo & Cualitativo \\
\hline 9 & Valderrama, C; Ortiz, H; Borrero, O. 2020 & $\begin{array}{c}\text { Análisis } \\
\text { Documental }\end{array}$ \\
\hline 10 & Vélez, A; Peñafiel, P; Heredia, M; Barreno, S; Chávez, J. 2019 & Cualitativo & Entrevistas \\
\hline 11 & Villegas, F; Llonch, J; López, P; Valenzuela, L. 2021 & Cuantitativo & Encuetas \\
\hline 12 & Maldovan, J. 2018 & Cualitativo & $\begin{array}{c}\text { Análisis } \\
\text { Documental }\end{array}$ \\
\hline
\end{tabular}

\section{Figura 3}

Porcentaje de enfoques metodológicos

\section{Enfoques Metodologicos}

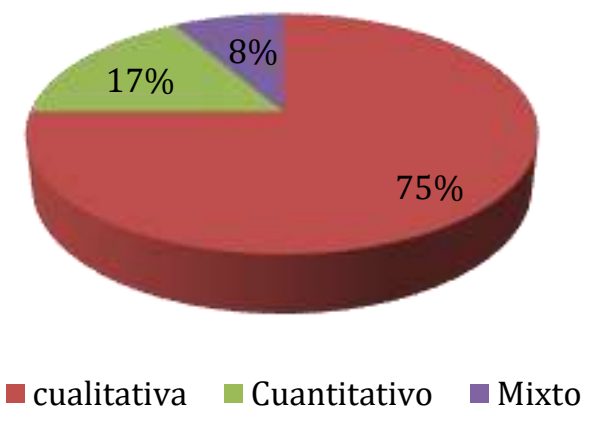


Por otro lado, sobre el instrumento para el recojo de información, podemos apreciar que la mayoría de las investigaciones, tres (3) de ellas, eligieron la encuesta. De estas, dos (2) fueron aplicadas a usuarios, uno (1) a recicladores. Del mismo modo, podemos notar que nueve (9) investigaciones optaron por instrumentos distintos como entrevistas (4) y análisis documental (5).

\section{Figura 4}

Porcentaje de Instrumentos de Recolección de Datos

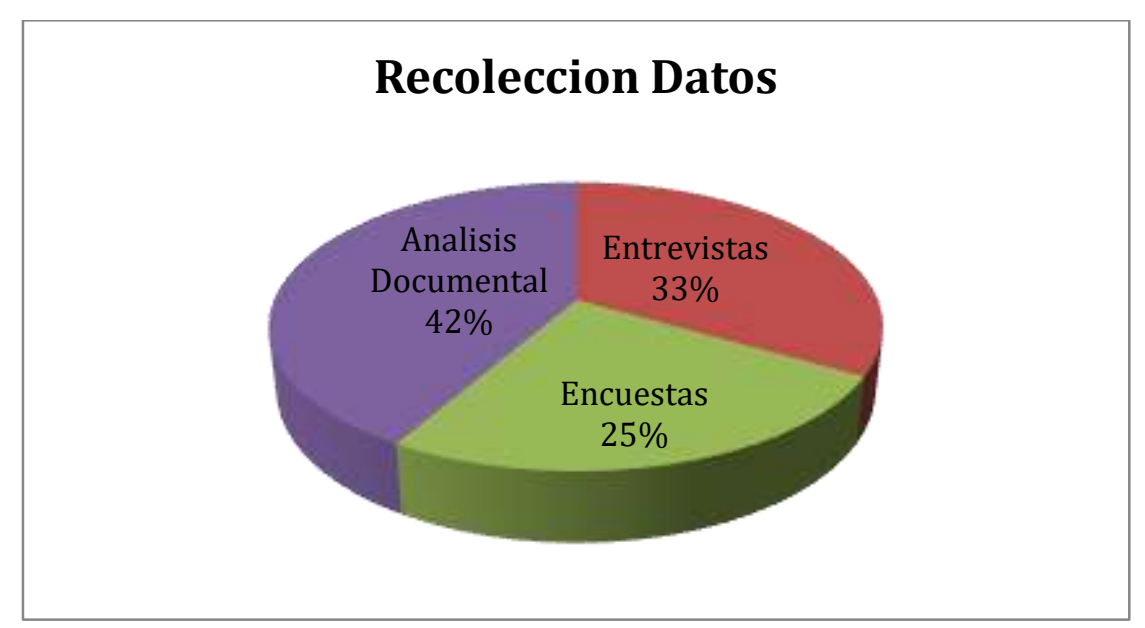

En las figuras puede apreciarse la preferencia de aplicar entrevistas al momento de recoger los datos, y ello se puede completar que la unidad de análisis se orienta a los recicladores y empresa privada, es decir, se recurre a la percepción, experiencia y opinión de uno de los actores del proceso en la Gestión de Residuos Sólidos, y en este caso es quien la recibe. En un segundo momento es orientado al punto de vista del sector gubernamental y por ultimo aplican los instrumentos a ambos.

\section{Conclusiones y Objetivos de los artículos revisados}

Según Vélez (2017) el excedente de los residuos sólidos actualmente, en Argentina y Latinoamérica se trabaja bajo un modelo económico en la compra y venta de residuos sin importar su origen, generando riqueza para la población pero al mismo tiempo hace que la informalidad sea un factor crítico en la generación de ingreso, es por ello, según Colorado y Echeverri (2019) los residuos sólidos (RS) en los países sub desarrollados tiene una relación directa entre la producción (PBI) y la generación de RS. Esta relación puede determinar los niveles de eficiencia productiva que pueden tener diferentes países, por ejemplo, cuando un país tiene una producción significativa pero la generación de sus residuos es mínima, los recursos se están utilizando de manera óptima. Por el contrario, 
una baja producción con una alta generación de residuos es un indicador de prácticas ineficientes.

Es así según Mendieta et al. (2020); Vélez et al. (2019) el proceso de recolección en la Parroquia Membrillo, Cantón Ecuador y en la Sociedad Waorani Gareno son deficientes, los individuos tienden a abrasar los residuos ocasionando un mal ambiental, y paralelamente deteriorando la calidad de vida. El proyecto de acción planteado es un instrumento que dejará la paz tanto ambiental como social, con tácticas que permiten mitigar las malas prácticas referentes a la manera de remover los desperdicios sólidos.

Según, Filipak et al. (2020) el reciclaje para muchos trabajadores es una última opción para sobrevivir dentro de las reglas sociales del mundo del trabajo, ya que proporciona a estas personas la posibilidad de trabajar. Esta apreciación los lleva a subestimar los riesgos y daños ocasionados por el trabajo, lo que impacta su relación con los servicios de salud. Es por ello, según Herrera et al. (2018) en su indagación plantea una revisión de las diversas metodologías disponibles para mejorar la gestión de los sistemas de recolección de desechos municipales en países en vías de desarrollo, en concordancia con los lineamientos del Programa de las naciones unidas. En dicha investigación se encontraron diversas herramientas, como por ejemplo un sistema informático que registra el flujo de materiales, por otro lado los estudios de caracterización y generación, los análisis de costos operativos y metodologías para reducir costos en las rutas de recolección, herramientas para la dirección estratégica del sistema de recolección de RS e instrumentos legales disponibles en torno a la temática de la recolección de RS, así mismo metodologías para medir la higiene y seguridad en operaciones implicadas y el impacto ambiental ocasionado en ellas, y finalmente se encontraron herramientas administrativas para el capital humano y equipos de recolección como vehículos y maquinarias adecuados para la selección y mantenimiento.

Es así según, Moreno (2019) en la actualidad, la disposición final de los residuos solidos provocados por la población de la metrópoli de Tacna Perú vienen llevando a cabo sus ocupaciones de segregación de residuos solidos, bajo el marco de la resolución de alcaldía $\mathrm{N}^{\circ}$ 0868-2010, donde se reconoce de manera oficial a las agrupación de recicladores, inscritos en la Superintendencia Nacional de los Registros Públicos y confirmaba su representatividad frente a la colectividad bajo el marco de la Ley del Reciclador. 
Así mismo sostiene Maldovan (2018), la formalización de la recolección de residuos sólidos urbanos en Buenos Aires Argentina es un camino que fortalece los diferentes conflictos que surgieron a raíz de los miles de recicladores. Esta creciente organización de los recicladores fue lo que les permitió avanzar en la adquisición de derechos, recursos y una mejor posición en materia de política ambiental, permitiendo a los recicladores mejorar sus condiciones de trabajo. Es por ello como dice Valderrama et al. (2020); Saidón (2019) es necesario diseñar políticas de gobierno hacia una economía informal contemplando las dimensiones financieras, ambiental y sociales con una mirada de largo plazo con una economía circular de reutilización y recuperación de RS. Que permita generar empleo formal sostenible con una modernización del sistema de gestión de residuos para aumentar la tasa de reciclaje y los ingresos basados en modelos de transición, que permitirá planificar su crecimiento laboral para mejorar las condiciones de seguridad y salud ocupacional mediante la obtención de vehículos de transporte y equipos de protección personal.

Pero, Villegas et al. (2021) advierte que no existe una normatividad clara y precisa de disposición de RS para la actividad privada como es el caso de las PYME en la Región de Chile encontrándose hallazgos como: La ausencia de una norma de desperdicio flexible y adecuada para las PYMES que considere sus características y limitaciones prácticas, la dificultades con el manejo y disponibilidad de materias primas sostenibles, la necesidad de apoyo directo para PYMES, reflejadas en herramientas como subsidios, ayudas financieras y asistencia técnica para abordar su gestión de residuos. Por tanto, es necesario un sentido holístico para abordar de forma eficaz la situación de la gestión de residuos de las PYME.

Finalmente según Valenzuela (2020) las ciudades de Medellín Colombia y Santiago Chile comparten muchas características. En Santiago, la gestión de residuos está privatizada y dominada por el capital transnacional; los servicios formales se concentran en áreas específicas según la rentabilidad o el exceso de recursos; el reciclaje está limitado por contratos a largo plazo; y los recicladores no pueden trabajar en algunos distritos de la ciudad. En cambio en Medellín, la gestión de residuos está a cargo de la empresa municipal de servicios públicos; los recicladores tienen derecho a recolectar materiales reciclables domésticos; sus servicios están repartidos por toda la ciudad; y parte de sus ingresos proviene de la redistribución de los ingresos fiscales municipales. 


\section{Innovación Tecnología en la Reutilización y Reciclaje de Residuos Sólidos.}

Según Vélez (2017) una vez que desafiemos a la sociedad de consumo a partir de la creación de otras formas de vida que no se funden en el consumismo, podremos, realmente transformar la realidad y las relaciones humanas, sin distinción en el consumo, logrando establecerse una estructura jerárquica de la misma vida, provocando la exclusión del sector menos consumidor de toda garantía y seguridad tanto social como política como sostiene Colorado y Echeverri (2019), la composición de Residuos Sólidos en Colombia incluye textiles y madera, lo que demuestra una oportunidad de innovación en estos sectores. Varias iniciativas de la industria y el mundo académico tienen como principales objetivos el reciclaje de residuos, para la fabricación de materiales ecológicos implementando la economía circular que desarrollo niveles bajos de $\mathrm{CO} 2$ fabricación de huella y la recolección de energía. Así mismo según Mendieta et al. (2020); Vélez, A. G. et al. (2019) la sociedad Cantón y Waorani Gareno Ecuador debe clasificar y guardar los residuos con un precio económico, como papel cartón, plástico, metal y desechos orgánicos, se deberá llevar al punto de acopio del gobierno en forma Independiente de la Descentralización Municipal de cantón Tena y paralelamente para el almacenaje de los residuos inorgánicos se facilitara contenedores adecuados bajo techo con una capacidad de almacenamiento suficiente para dos (2) meses. En cuanto a la parte orgánica, se indica que la sociedad asigne una persona quien se encargará de la preparación del compostaje. Y según Filipak et al. (2020) el trabajo realizado del reciclador diario es arduo, con cargas pesadas, sin un horario fijo, con exigencias físicas y con poca rentabilidad económica. Este trabajo, por su autonomía, horario flexible y relación directa entre producción y ganancia económica, es percibido como un servicio gratificante, divertido y en ocasiones visto como el mayor placer en la vida de los trabajadores. Para el traslado de los residuos sólidos utilizan un carro grande o carreta sin motor, el motor son los recicladores que lo empujan con mucho esfuerzo.

Así mismo según Herrera et al. (2018) en Ecuador hasta el año 1998 no se contaba con instrumentos legales de gestión de RS, sin embargo en la actualidad hay normativas orientadas a la conservación del medio ambiente y garantizar el derecho de los pobladores a vivir en un ambiente sano y ecológicamente equilibrado bajo un plan nacional del buen vivir y la normatividad aprobada por el ministerio del ambiente, creada en año 2002, y el Programa Nacional para la Gestión Integral de Desechos Sólidos 2010, sin embargo, en 
el contexto local el reciclaje constituye una propuesta real para mejorar el aprovechamiento de los recursos, la limpieza del entorno y la generación de ingresos en diversos grupos vulnerables de la sociedad, especialmente en la ciudad de Ibarra-Ecuador, siendo los materiales mayormente colectados el hierro, acero, el cartón corrugado, el plástico y otros como el caucho, chatarra electrónica, madera y textiles, etc. En dicho estudio se pudo evidenciar y comprobar la existencia de inadecuadas condiciones laborales en los recicladores, con presencia de trabajo infantil dentro de la recolección de desechos sólidos. En cuanto a los equipos de protección personal, son deficientes y los recicladores laboran en forma independiente e informal que nos le permite aprovechar los beneficios que podrían gozar como trabajador bajo asociación. Según Moreno (2019) actualmente, la disposición final de los desechos generados por la población de los distritos de Tacna, está en el Botadero Municipal de dicha localidad, favoreciendo este marco normativo a la regulación de las actividades de los trabajadores del reciclaje, orientado a la protección, capacitación y promoción del desarrollo social y laboral, promoviendo su formalización, sociedad y contribuyendo a la mejora en el desempeño ecológicamente eficiente de los residuos rígidos en el territorio, bajo un plan de gestión de residuos sólidos con desarrollo sostenible. Pero según Maldovan (2018) la formalización de la recolección de residuos sólidos urbanos en buenos Aires, ha permitido la creciente organización de los recicladores avanzar en la adquisición de derechos y recursos; y así mismo una mejor posición en materia de política ambiental y el acceso a beneficios que permitieron a los recicladores mejorar sus condiciones de trabajo, a pesar que el proceso de recolección de residuos sigue siendo con carretajes en forma artesanal. Y según (Valderrama, C. et al., 2020; Saidón, 2019), los propietarios de las empresas de reciclaje (Ciudad de Neiva) tienen títulos de educación primaria (60\%) y secundaria (40\%). La mayor parte del sector empresarial ha estado operando durante más de cinco (5) años (50\%) y entre 1 y 5 años (40\%) en esas condiciones. La mayoría de las empresas de reciclaje en Neiva son de propiedad familiar, donde la mayoría de ellas tienen entre 1 y 3 empleados (50\%). Sin embargo haciendo un análisis más profundo sobre el uso de rellenos sanitarios más modernos, el descuento de los posibles ingresos por recuperación de materiales o energía, el mayor ahorro se daría con la termo-valorización, luego con la tecnología de Tratamiento Mecánico-Biológico (TMB), la recuperación energética, con captación de gas y electricidad permitiría al relleno sanitario un manejo adecuado de los 
RS. Por otra parte, en términos de ingresos, el compost aeróbico y la digestión anaeróbica permiten vender abono orgánico como sub-producto, siendo los retornos más rápidos y mayores en la digestión anaeróbica, que también permite ahorros en la producción de gas. Finalmente, según Villegas et al. 2021) los gestores de las PYMES sí consideraron establecer diferentes iniciativas e incentivos para reducir la generación de residuos en sus actividades, en función de su interacción diaria con sus grupos de interés. En primer lugar, incentivos económicos a través de reducciones de impuestos y/o ayuda financiera para invertir en el proceso de producción más limpia. En segundo lugar, el desarrollo de redes de colaboración entre PYMES e instituciones públicas en la materia. En este sentido, si las normas pudieran ser más adaptativas y flexibles, las PYMES podrían generar ventajas competitivas y generar beneficios sostenibles. según Valenzuela (2020) desde el 2015 la mayoría de los municipios del Área Metropolitana de Santiago de Chile depositan sus desechos en dos vertederos o relleno sanitarios de propiedad de empresas transnacionales. Uno de ellos (empresa hispanoamericana Urbaser Danner Group). Y el otro es (empresa francesa Veolia) y un tercer vertedero, es propiedad del holding chileno Consorcio Santa Marta. Por otra parte el sistema de deposición en la ciudad colombiana se basa en el trabajo que realizan las cooperativas de reciclaje, que incluyen 3.662 registrados.

\section{CONCLUSIÓN Y RECOMENDACIONES}

En conclusión, tras el esfuerzo por integrar los resultados analizados en este trabajo, parece bastante fundamental asegurar que la reutilización y reciclaje de los residuos rígidos en América Latina todavía está en proceso de aprendizaje en la Administración de Residuos Sólidos con un proceso de producción lineal que no asegura minimizar el consumo discriminado de bienes y servicios, prevaleciendo en las Instituciones Gubernamentales y No Gubernamentales de América Latina. Se requiere destacar que las zonas de esta parte del conjunto de naciones en el año 2015 aprobaron las metas de Desarrollo Sustentable conjuntamente con la ONU para colocar fin a la pobreza, defender el mundo y asegurar que para el año 2030 toda la gente gocen de tranquilidad y prosperidad. No obstante poco se ha hecho en la probabilidad de orientar a una transición, hacia una economía circular a partir de una visión del parlamento Europeo y la Comisión Económica para América Latina y el Caribe con el objeto de llevar a cabo un nuevo proceso de Administración de Residuos sólidos enmarcado en un nuevo modelo económico y social que posibilite reducir el consumo y el desperdicio de materias primas, 
agua y fuentes de energía; y que se mantengan en el sistema económico en el lapso de tiempo más extenso, teniendo presente el medio ambiente y la sostenibilidad, (Rincón et al., 2021; Tello, 2018).

\section{REFERENCIAS BIBLIOGRAFICAS}

Barros, M. V., Salvador, R., do Prado, G. F., de Francisco, A. C., \& Piekarski, C. M. (2021). Circular economy as a driver to sustainable businesses. Cleaner Environmental Systems, 2(June), 100006. https://doi.org/10.1016/j.cesys.2020.100006

Colorado, H. A., \& Echeverri-Lopera, G. I. (2020). The solid waste in Colombia analyzed via gross domestic product: Towards a sustainable economy. Revista Facultad de Ingeniería Universidad de Antioquia, (96), 51-63. http://www.scielo.org.co/scielo.php?pid=S0120$62302020000300051 \&$ script=sci_arttext\&tlng=en

Comision, Europea. (2018). Sobre un marco de seguimiento para la economía circular \{SWD(2018) 17 final $\}$. Comision Europea.

https://op.europa.eu/es/publication-detail/-/publication/d7c37d9d-fabf-11e7-b8f501aa75ed71a1

Corona, B., Shen, L., Reike, D., Rosales, J., \& y Worrell, E. (2019). Towards sustainable development through the circular economy-A review and critical assessment on current circularity metrics. Resources, Conservation and Recycling, 151(September 2019), 104498. https://doi.org/10.1016/j.resconrec.2019.104498

De La Cuesta, M; Pardo, E. (2020). Transicion a la economia circular. Fundacion ICO y UNED, 1(Junio), 1-105. https://www.fundacionico.es/economia-circular/

Ekins, P., Domenech, T., Drummond, P., Bleischwitz, R., Hughes, N., \& Lotti, L. (2019). The Circular Economy: What, Why, How and Where. Managing environmental and energy transitions for regions and cities. Background Background Paper for an OECD/EC Workshop on 5 July 2019 within the Workshop Series "Managing Environmental and Energy Transitions for Regions and Cities” Paris, 1(July), 189. http://ceb.allconfs.org/img/20200406/200406_1854188.pdf

Filipak, A; Stefanello, S; Mirodi, J; Hunzcker, M. H. y Dantas, D. V. (2020). Artículos "O motor é a gente mesmo": cuidado em saúde dos trabalhadores da reciclagem. Revista Interface, 24(1), 1-15. https://doi.org/10.1590/ Interface.190472 
Goodwin, E; Novak, M; Haigh, L; Birliga, A; Wildi, D; Wynaendts, S. y Dufourmont, J. (2021). Key Elements of the Circular Economy. Circlen Economy, 1(February), 1-37. https://www.tandfonline.com/doi/full/10.1080/21681015.2016.1172124

Graziani, P. (2018). Economia Circular e innovacion tecnologica en residuos solidos Oportunidades en America

Latina. http://scioteca.caf.com/handle/123456789/1247

Grijalva, P. K., Cornejo, G. E., Gómez, R. R., REal, K. P., \& Fernandez, A. (2019). Herramientas colaborativas para revisiones sistemáticas. Revista Espacios, 40(5), 1-9. https://www.revistaespacios.com/a19v40n25/a19v40n25p09.pdf

Herrera, I. D; Herrera, E.P; Collaguazo, G; Lorente, L. L; Saraguro, R. V. (2018). REVISIÓN DE BUENAS PRÁCTICAS EN EL SISTEMA DE RECOLECCIÓN DE RESIDUOS: UN ANÁLISIS PARA LA IMPLEMENTACIÓN LOCAL. DELOS Desarrollo Local Sostenible, 11(32), 1-25. https://www.eumed.net/rev/delos/32/israel.html

Maldovan, J. (2018). Environmental Protection, Work, and Social Inclusion: Formalizing the Recycling of Urban Solid Waste in Buenos Aires. Latin American Perspectives, 45(1), 91-107. https://doi.org/10.1177/0094582X17730372

Mendieta-Vivas, R. J., Giler-Sarmiento, J. A., Menéndez-Cevallos, C. Y., \& MacíasChila, R. R. (2020). Estudio sobre el manejo de desechos sólidos del área urbana en la parroquia Membrillo, cantón Bolívar. Dominio de las Ciencias, 6(3), 282309. https://www.dominiodelasciencias.com/ojs/index.php/es/article/view/1285

Moher, D., Shamseer, L., Clarke, M., Ghersi, D., Liberati, A., Petticrew, M., Shekelle, P., Stewart, L. A., Estarli, M., Barrera, E. S. A., Martínez-Rodríguez, R., Baladia, E., Agüero, S. D., Camacho, S., Buhring, K., Herrero-López, A., Gil-González, D. M., Altman, D. G., Booth, A., ... Whitlock, E. (2016). Preferred reporting items for systematic review and meta-analysis protocols (PRISMA-P) 2015 statement. Revista Espanola de Nutricion Humana y Dietetica, 20(2), 148-160. https://doi.org/10.1186/2046-4053-4-1

Moreno Salazar Calderón, K. A. B. (2019). Una mirada a las prácticas de reciclaje: El caso de una asociación de recicladores en Tacna, Perú. Revista Estudios del Desarrollo Social: Cuba y América Latina, 7(3). http://scielo.sld.cu/scielo.php?pid=S2308- 
$01322019000300022 \&$ script $=$ sci_arttext\&tlng=en

Patwa, N., Sivarajah, U., Seetharaman, A., Sarkar, S., Maiti, K., \& Hingorani, K. (2021). Towards a circular economy: An emerging economies context. Journal of Business Research, 122, 725-735. https://doi.org/10.1016/j.jbusres.2020.05.015

Prieto, V., Jaca, C., \& Ormazabal, M. (2018). Towards a consensus on the circular economy. Journal of Cleaner Production, 179(1), 605-615. https://doi.org/10.1016/j.jclepro.2017.12.224

Rincón, J., Ormazábal, M., Álvarez, M. J., \& Jaca, C. (2021). Advancing circular economy performance indicators and their application in Spanish companies. Journal of Cleaner Production, 279(January), 123605. https://doi.org/10.1016/j.jclepro.2020.123605

Romero, D., \& Rossi, M. (2017). Towards Circular Lean Product-Service Systems. Procedia CIRP, 64, 13-18. https://doi.org/10.1016/j.procir.2017.03.133

Rondón, E., Szantó, M., Pacheco, J. F., Contreras, E., \& Gálvez, A. (2016). Guía general para la gestión de residuos sólidos domiciliarios. Manuales de La CEPAL, 2, 209. http://hdl.handle.net/11362/40407

Saidón, M. (2019). Análisis de alternativas para la gestión actual de residuos en la Ciudad de Buenos Aires a partir de distintos criterios. Gestión y Ambiente, 22(1), 115127. https://doi.org/10.15446/ga.v22n1.78338

Sánchez, M. del P., Cruz, J. G., \& Maldonado, P. C. (2020). Gestión de residuos sólidos urbanos en América Latina: un análisis desde la perspectiva de la generación. Revista Finanzas y Política Económica, 11(2), 321-336. https://doi.org/10.14718/revfinanzpolitecon.2019.11.2.6

Sanmartin, G. S; Zhigue, R. A; Alaña, T. P. (2016). El Reciclaje: Un nicho de innovacion y emprendimiento con enfoque ambientalista. Revista Científica Universidad y Sociedad, 8(3), 150. http://rus.ucf.edu.cu/

Schröder, P; Albaladejo, M; Alonso Ribas, P; MacEwen, M y Tilkanen, J. (2020). Liberando la economía circular en América Latina y el Caribe. CHATHAM HOUSE, 73. https://www.chathamhouse.org/sites/default/files/2021-01/2021-0113-spanish-circular-economy-schroder-et-al.pdf

Tello P, Campani D, S. M. (2018). Gestion Integral de Residuos Solidos Urbanos. https://aidisnet.org/wp-content/uploads/2019/08/GESTION-INTEGRAL-DE- 


\section{RESIDUOS-SOLIDOS-URBANOS-LIBRO-AIDIS.pdf}

Turcott, D. (2018). Sistema de Indicadores para la Evaluacion Integral y Control de la Gestion de Residuos Municipales. Universidad de Cantabria Pais Vasco, 1-449. https://web.unican.es/

Valderrama, C., Ortiz, J. D., \& Unidos, M. M. y Borrero, O. I. (2020). Caso de estudio Neiva , Colombia Análisis del sector reciclador en ciudades intermedias . Estudio de caso municipio de Neiva, Colombia. Ingeniería y Competitividad Revista $\begin{array}{llll}\text { Científica } & y & \text { Tecnológica, } & \text { 22(2), }\end{array}$ https://revistaingenieria.univalle.edu.co/index.php/ingenieria_y_competitividad/ article/view/6252

Valenzuela, N. (2020). Waste Political Settlements in Colombia and Chile: Power, Inequality and Informality in Recycling. Development and Change, 51(4), 10981122. https://doi.org/10.1111/dech.12591

Vélez, A. G., Arcos, P. A. P., Rengifo, M. G. H., Ubilluz, S. N. B., \& Espinoza, J. F. C. (2019). Propuesta de sistema de gestión de residuos sólidos domésticos en la comunidad Waorani Gareno de la Amazonía Ecuatoriana. Revista Ciencia y Tecnología, 12(2),

$33-45$. https://dialnet.unirioja.es/servlet/articulo?codigo=7563002

Vélez, J. (2017). El excedente de los residuos sólidos: entre metafísica, biopolítica y gobierno. Estudios de Filosofía Práctica e Historia de Las Ideas, 19(1), 1-12. https://dialnet.unirioja.es/servlet/articulo?codigo $=6535451$

Villegas, F. J; Llonch, J; López, P; Valenzuela, L. (2021). Waste Management. The Disconnection between Normative and SMEs Reality. Sustainability, 13(1787), 1-21. https://doi.org/10.3390/su13041787

Yáñez, P. P. (2021). Viabilidad de la economía circular en países no industrializados y su ajuste a una propuesta de economías transformadoras. Un acercamiento al escenario latinoamericano. CIRIEC-Espana Revista de Economia Publica, Social y Cooperativa, 101, 289-323. https://doi.org/10.7203/CIRIEC-E.101.15979 